\title{
Injection of Oort Cloud comets: the fundamental role of stellar perturbations
}

\author{
Hans Rickman • Marc Fouchard • \\ Christiane Froeschlé • Giovanni B. Valsecchi
}

Received: 30 November 2007 / Revised: 5 March 2008 / Accepted: 11 April 2008 /

Published online: 12 June 2008

(C) Springer Science+Business Media B.V. 2008

\begin{abstract}
We present Monte Carlo simulations of the dynamical evolution of the Oort cloud over the age of the Solar System, using an initial sample of one million test comets without any cloning. Our model includes perturbations due to the Galactic tide (radial and vertical) and passing stars. We present the first detailed analysis of the injection mechanism into observable orbits by comparing the complete model with separate models for tidal and stellar perturbations alone. We find that a fundamental role for injecting comets from the region outside the loss cone (perihelion distance $q>15 \mathrm{AU}$ ) into observable orbits $(q<5 \mathrm{AU})$ is played by stellar perturbations. These act in synergy with the tide such that the total injection rate is significantly larger than the sum of the two separate rates. This synergy is as important during comet showers as during quiescent periods and concerns comets with both small and large semi-major axes. We propose different dynamical mechanisms to explain the synergies in the inner and outer parts of the Oort Cloud. We find that the filling of the observable part of the loss cone under normal conditions in the present-day Solar System rises from $<1 \%$ for $a<20000 \mathrm{AU}$ to about $100 \%$ for $a \gtrsim 100000 \mathrm{AU}$.
\end{abstract}

H. Rickman

PAN Space Research Center, Bartycka 18A, 00-716 Warszawa, Poland

H. Rickman

Uppsala Astronomical Observatory, Box 515, 75120 Uppsala, Sweden e-mail: hans@astro.uu.se

M. Fouchard $(\varangle)$

LAL-IMCCE/USTL, 1 Impasse de l'observatoire, 59000 Lille, France

e-mail: fouchard@imcce.fr

C. Froeschlé

Observatoire de la Côte d'Azur, UMR 6202, Bv. de l'Observatoire,

B.P. 4229, 06304 Nice cedex 4, France

e-mail: froesch@obs-nice.fr

G. B. Valsecchi

INAF-IASF, via Fosso del Cavaliere 100, 00133 Roma, Italy

e-mail: giovanni@iasf-roma.inaf.it 
Keywords Galactic tide perturbations - Monte Carlo simulation · Long period comets · Oort Cloud

\section{Introduction}

When analysing the distribution of original inverse semi-major axes of long-period comets, Oort (1950) concluded that the near-parabolic spike of this distribution reveals a distant reservoir of comets (the 'Oort Cloud'). His favoured mechanism of injection of comets from this reservoir into observable orbits (i.e., with small perihelion distances) was the passage of stars in the vicinity of the reservoir, whereby the long-term reshuffling of angular momenta would ensure a continuous infeed into the innermost part of the Solar System.

Until the 1980s stellar perturbation was the only mechanism considered when issues concerning the injection of comets from the Oort Cloud were discussed (e.g., Rickman 1976; Weissman 1979; Fernández 1980; Hills 1981; Remy and Mignard 1985). However, by that time it became clear that the tidal action of the Galaxy as a whole must also have an important influence-especially the part corresponding to the $z$-dependent disk potential (Heisler and Tremaine 1986). This was verified by noting that the Galactic latitudes of perihelia of new Oort Cloud comets have a double-peaked distribution that is characteristic of the disk tide (Delsemme 1987).

An important paper by Duncan et al. (1987) treated the formation of the Oort Cloud and showed that the characteristic time scale for changing the perihelion distances, independent of the semi-major axis, is shorter for the Galactic disk tide than for the stellar perturbations. This has been verified by later analytic work, e.g., by Fernández (2005), and further numerical simulations of Oort Cloud evolution (e.g., Heisler et al. 1987) have given support to the dominance of Galactic tides for comet injection.

Consequently, stellar perturbations have come to be practically neglected as a source of comet injection-except when discussing "comet showers" (Hills 1981) arising from rare stellar passages through the dense, inner parts of the Oort Cloud. The importance of stellar perturbations for randomizing the orbital distribution of the Oort Cloud and thus keeping the relevant infeed trajectories of the disk tide populated over long time scales has been realized (see Dybczyński 2002 and references therein), but the actual injection is often seen as due only to the tide. Hence it should be limited to semi-major axes large enough for the tidal perturbation to bring the cometary perihelion at once from outside the "Jupiter-Saturn barrier" (i.e., perihelion distance $q \gtrsim 15 \mathrm{AU}$ ) into the observable region ( $q<5 \mathrm{AU}$ ). The result is that one expects new comets to have $a_{\text {ori }} \gtrsim 3 \times 10^{4}$ AU (Bailey and Stagg 1988; Levison et al. 2001; Fernández 2005).

On the other hand, some recent papers indicate that this picture may have problems. The fractional population of the observable region - if fed only by Galactic tides-is small enough, and the orbital periods long enough, that the estimated total population of the Oort Cloud may be uncomfortably large (Charnoz and Morbidelli 2007). And in addition, when non-gravitational effects are included into orbit determinations for new Oort Cloud comets (Królikowska 2006), the resulting original orbits tend to be of shorter periods, having smaller semi-major axes-often much smaller than $3 \times 10^{4} \mathrm{AU}$.

Meanwhile, we have developed fast and accurate methods to treat both the Galactic tides (Breiter et al. 2007; Fouchard et al. 2007) and stellar perturbations (Rickman et al. 2005) in Monte Carlo simulations of Oort Cloud dynamics. This has allowed us to perform calculations, to be presented here, where the cloud is represented by as many as $10^{6}$ sample comets and integrated over a time exceeding the age of the Solar System. This amounts to 
$5 \times 10^{15}$ comet-years of individual evolutions (or only slightly smaller due to the loss of comets during the simulation), which is much more than in all previous long-term Oort Cloud simulations-e.g., $3 \times 10^{13}$ comet-years for Duncan et al. (1987), $4 \times 10^{14}$ comet-years for Mazeeva (2006), and $\sim 4 \times 10^{13}$ comet-years for Emel'yanenko et al. (2007) who used cloning. Heisler (1990) simulated $\simeq 7 \times 10^{15}$ comet-years but only thanks to extensive cloning during the course of the simulation. In fact her long-term simulations $(4.5 \mathrm{Gyr})$ concerned only $\simeq 10^{4}$ "tokens", i.e., comets actually followed, while these were meant to represent $\simeq 150$ times as many comets by means of cloning.

Our work is the first to study the mechanism of injection of comets from the Oort Cloud over the age of the Solar System by simulating and comparing different dynamical models. The reason why models involving both the Galactic tide and stellar perturbations gave a much higher flux of injected comets than those involving only stars (Heisler et al. 1987; Heisler 1990) was never clarified, since comparisons with models involving only the tide were not made. In the present paper we concentrate on a comprehensive comparison of combined and separate models, thus describing and analysing for the first time the synergy effect of Galactic tide and stars.

We also take special care to define correctly the encounter velocities in our sample of passing stars, thereby arriving at somewhat larger values than those used previously. Finally, we study the relative filling of the observable part of the loss cone and the distribution of inverse semi-major axes of the injected comets. These studies are, however, only preliminary, since our current simulations do not include planetary perturbations, and thus we cannot account for those comets that arrive into the observable region after having "diffused" across the Jupiter-Saturn barrier in several revolutions.

Our calculations are presented in Sect.2, and in Sects.3-5 we describe our results in terms of the distribution of injection times into the inner planetary system, the flux of new, observable comets as a function of time, and the distributions of inverse semi-major axis and Galactic latitude of perihelion as well as loss cone filling at representative epochs. In Sect. 6 we discuss the results and summarize our conclusions.

\section{Calculations}

As a simplifying assumption, we consider the Oort Cloud to have been formed instantaneously at a given epoch, and that its orbital distribution was isotropic to begin with. Thus the initial conditions are chosen with flat distributions of $\cos i_{o}, \omega_{o}, \Omega_{o}$ and $M_{o}$ (we use common notations for the orbital elements, and the angles may be defined with respect to an arbitrary frame of reference). We consider a thermalized initial state of the cloud, where the semi-major axes $\left(a_{o}\right)$ are chosen in the interval $3 \times 10^{3}<a_{o}<1 \times 10^{5}$ AU with a probability density $\propto a_{o}^{-1.5}$ (Duncan et al. 1987). The initial eccentricities $\left(e_{o}\right)$ are chosen with a density function $\propto e_{o}$ in such a way that the perihelia are outside the planetary system $(q>32 \mathrm{AU})$. We thus initialise $1 \times 10^{6}$ comets.

The Galactic parameters used for calculating the tidal effects are the same as described in earlier papers (Fouchard et al. 2007). The most important one for comparison with other investigations is the mid-plane disk density, which we take as $0.1 M_{\odot} \mathrm{pc}^{-3}$ (Holmberg and Flynn 2000). This is in agreement with Emel'yanenko et al. (2007), while Heisler (1990) used $0.18 M_{\odot} \mathrm{pc}^{-3}$ (Bahcall 1984).

The simulation runs with a predefined set of 197,906 stellar encounters, occurring at random times during a lapse of $t_{\max }=5 \times 10^{9}$ years, with random solar impact parameters up to $d_{\max }=4 \times 10^{5} \mathrm{AU}$, and with random stellar masses and velocities. Our procedure for creating each of these encounters is as follows. Let $\xi$ denote a stochastic, random number 
Table 1 Stellar parameters

\begin{tabular}{lllllll}
\hline Type & Mass $\left(M_{\odot}\right)$ & Enc. freq. & $v_{\odot}(\mathrm{km} / \mathrm{s})$ & $\sigma_{*}(\mathrm{~km} / \mathrm{s})$ & $\langle V\rangle(\mathrm{km} / \mathrm{s})$ & $\sigma_{V}(\mathrm{~km} / \mathrm{s})$ \\
\hline B0 & 9 & 0.005 & 18.6 & 14.7 & 24.6 & 6.7 \\
A0 & 3.2 & 0.03 & 17.1 & 19.7 & 27.5 & 9.3 \\
A5 & 2.1 & 0.04 & 13.7 & 23.7 & 29.3 & 10.4 \\
F0 & 1.7 & 0.15 & 17.1 & 29.1 & 36.5 & 12.6 \\
F5 & 1.3 & 0.08 & 17.1 & 36.2 & 43.6 & 15.6 \\
G0 & 1.1 & 0.22 & 26.4 & 37.4 & 49.8 & 17.1 \\
G5 & 0.93 & 0.35 & 23.9 & 39.2 & 49.6 & 17.9 \\
K0 & 0.78 & 0.34 & 19.8 & 34.1 & 42.6 & 15.0 \\
K5 & 0.69 & 0.85 & 25.0 & 43.4 & 54.3 & 19.2 \\
M0 & 0.47 & 1.29 & 17.3 & 42.7 & 50.0 & 18.0 \\
M5 & 0.21 & 6.39 & 23.3 & 41.8 & 51.8 & 18.3 \\
wd & 0.9 & 0.72 & 38.3 & 63.4 & 80.2 & 28.2 \\
gi & 4 & 0.06 & 21.0 & 41.0 & 49.7 & 17.5 \\
\hline
\end{tabular}

The types are mostly MK types for main sequence stars; 'wd' indicates white dwarfs, and 'gi' indicates giant stars. The encounter frequencies are given in number per Myr within $1 \mathrm{pc}$. The following two columns list the solar apex velocity with respect to the corresponding type, and the spherical Maxwellian velocity dispersion. The last two columns give the mean heliocentric encounter velocity and its standard deviation according to our results

with a uniform probability distribution on the interval $[0,1]$. The solar impact parameter is chosen as $d=\xi_{d}^{1 / 2} \times d_{\text {max }}$, and the time of the encounter (specifically, the time of the star's perihelion passage) is $t=\xi_{t} \times t_{\max }$. The direction of stellar motion with respect to the Sun is defined in terms of Galactic latitude and longitude $(b, \ell)$ such that $\sin b=2 \xi_{b}-1$ and $\ell=\xi_{\ell} \times 2 \pi$, i.e., it has an isotropic distribution. The point at which the initial stellar velocity cuts the orthogonal impact plane is situated on a circle of radius $d$ around the Sun, and its location is defined by an azimuthal angle (a) such that $a=\xi_{a} \times 2 \pi$.

Next we choose the type of the star. We use 13 categories as in Rickman et al. (2004) with parameters listed in Table 1. To each category we associate one value of the stellar mass. These masses are generally taken as those of the archetypal spectral classes along the main sequence according to Allen (1985). However, in contrast to our earlier investigations, we replace the archetypal mass of $18 M_{\odot}$ for B0 stars by a weighted average of $9 M_{\odot}$, considering that the less massive, later types (B2, B5) are much more common than the earlier ones. The relative encounter frequencies $f_{i}$ of Table 1 are taken from García-Sánchez et al. (2001), where they were derived from the respective products of number density and mean velocity, $n_{i}\left\langle v_{i}\right\rangle$. A random number $\xi_{i}$ is used to pick a stellar category $i$ with the probability $f_{i} / \sum f_{i}$.

Finally, we choose the speed of the stellar motion in the following way. The velocity dispersions $\left(\sigma_{* i}\right)$ listed in Table 1 are taken from García-Sánchez et al. (2001), and they correspond to the semi-axes of the velocity ellipsoids $\left(\sigma_{u i}, \sigma_{v i}, \sigma_{w i}\right)$ listed by Mihalas and Binney (1981) using: $\sigma_{* i}^{2}=\sigma_{u i}^{2}+\sigma_{v i}^{2}+\sigma_{w i}^{2}$. For the peculiar velocity $\left(v_{*}\right)$ of a star with respect to its LSR, we use a spherical Maxwellian by taking $\eta_{u}, \eta_{v}$ and $\eta_{w}$ as three random numbers, each with a Gaussian probability distribution with expectance 0 and variance 1 , and computing $v_{*}=\sigma_{* i}\left\{\left(\eta_{u}^{2}+\eta_{v}^{2}+\eta_{w}^{2}\right) / 3\right\}^{1 / 2}$. The star's heliocentric velocity is found by combining the vector $\mathbf{v}_{*}$ with the Sun's peculiar velocity with respect to the star's LSR ("apex velocity") $\mathbf{v}_{\odot}$, whose absolute value is listed in Table 1 for each stellar category. We assume a random relative orientation of the two vectors and thus compute:

$$
V=\left\{v_{\odot i}^{2}+v_{*}^{2}-2 v_{\odot i} v_{*} \cdot C\right\}^{1 / 2}
$$

where $C=\cos \theta$ is taken as $C=2 \xi_{C}-1$, and $\theta$ is the angle between the two vectors. 
Within each stellar category we have to account for the fact that the contribution to the encounter flux is proportional to $V$. Thus we define a constant, large velocity $V_{0 i}=v_{\odot i}+3 \sigma_{* i}$ for each category, such that $V$ is always smaller than $V_{0 i}$, and we take a new random number $\xi_{V}$ and keep the value just found for $V$, if $\xi_{V}<V / V_{0 i}$. Otherwise we repeat the computation of $V$ until the $\xi_{V}$ condition is fulfilled. This procedure was not followed in our previous investigations, leading to underestimates of the average stellar velocities. Further underestimates were caused by programming errors, and we caution the reader that the stellar velocities in Rickman et al. (2004, 2005) were systematically too small. This is clearly seen by comparing Fig. 1 of Rickman et al. (2005) with the data in Table 1, which yield a mean velocity of $53 \mathrm{~km} / \mathrm{s}$ with a dispersion of $\simeq 20 \mathrm{~km} / \mathrm{s}$.

A few comments on the mean stellar encounter velocity are in order. The average peculiar velocity of stars in the solar neighbourhood is $\simeq 40 \mathrm{~km} / \mathrm{s}$. This value was given by Hut and Tremaine (1985), and combining it in quadrature with a typical solar apex velocity of $23 \mathrm{~km} / \mathrm{s}$ for the most common stellar categories (Table 1), one gets a mean heliocentric velocity of $\simeq 46 \mathrm{~km} / \mathrm{s}$. Heisler et al. (1987) were the first to introduce the flux-weighting into the selection of random velocities, but they neglected the solar apex velocity. In fact, their flux-weighting was somewhat different from ours, because they considered only one of the three velocity components, namely, the radial heliocentric velocity. But the encounter flux is sensitive to the velocity with respect to the impact plane, i.e., the full speed of the star, instead of just the radial component. We have found that this difference has only a small effect on the resulting mean velocity, but we mention it for the sake of completeness. In both cases, we find that the weighting raises the mean velocity by $6-7 \mathrm{~km} / \mathrm{s}$. This explains our mean velocity of $53 \mathrm{~km} / \mathrm{s}$ as resulting from including both the solar motion and the flux-weighting procedure. Finally, let us compare with the mean encounter velocity of $\simeq 46 \mathrm{~km} / \mathrm{s}$ in García-Sánchez et al. (2001). This resulted from a list of 92 stellar encounters within $5 \mathrm{pc}$ and $1 \mathrm{Myr}$ of the present, compiled with the aid of Hipparcos data, but the authors showed that there was a serious bias against faint absolute magnitudes in this sample, affecting all stars with $M_{V}>4$. Thus, the stars with the highest velocities were essentially lacking, and the resulting mean velocity is that of the inherently brighter, slower moving stars.

Comparing with other investigators, we note that both Heisler (1990); Mazeeva (2006) and Emel'yanenko et al. (2007) based their stellar encounter frequencies on the analysis by Heisler et al. (1987), who ignored the solar motion - thereby underestimating the relative frequency of encounters with massive stars that have small velocity dispersions - and neglected the contribution of the massive giants. Our encounter sample contains as much as $3.5 \%$ of massive stars, i.e., the B0, A0, A5, F0, F5 and 'gi' categories in Table 1 with an average mass of $2.3 M_{\odot}$, while counting the stars in the absolute magnitude range that corresponds to this average mass in Heisler et al. (1987), one arrives at $<1 \%$ of the total encounter frequency. Since the massive stars have an average $M / V$ ratio $\sim 10$ times larger than the red dwarf stars that dominate the sample, each such star will affect $\sim 100$ times as many comets. Hence one easily realizes that in our case a large fraction of the total stellar effect comes from the massive star category that is downplayed by the other investigators. This, to some extent, compensates for two other effects that make the stellar perturbations less efficient in our simulation. One is the larger encounter velocities, as already described, and the other is the total encounter frequency within $1 \mathrm{pc}$, which in our case is 10.5 per Myr, while for the others it is 13.1 per Myr.

Our calculations of the heliocentric impulse imparted to the comet (at time $t$ ) are done using the Sequential Impulse Approximation, which guarantees a good accuracy at a low cost of computing time (Rickman et al. 2005). During the simulation we keep track of all the perihelion passages with their $q$ values. At each perihelion a decision is taken about which 

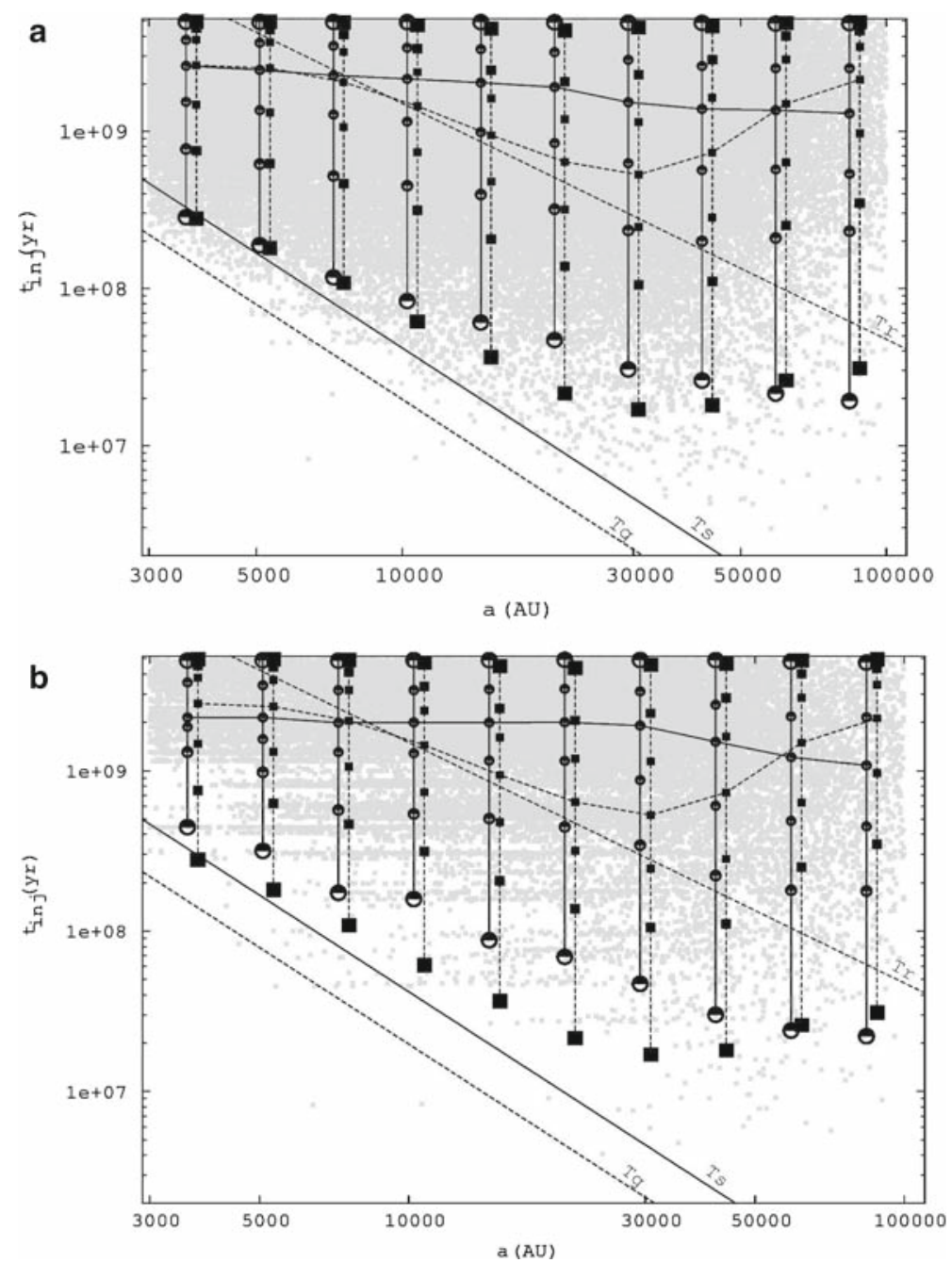

Fig. 1 Injection time versus initial semi-major axis. The semi-major axis range is split into 10 equal intervals of $\log a$. For each interval we plot different statistical parameters characterizing the distribution of injection times, as explained in the text. The dotted line labelled $T_{g}$ corresponds to the tidal torquing time shown in Fig. 2 of Duncan et al. (1987), $T_{S}$ to the corresponding stellar torquing time, and $T_{r}$ to the period of $\left(q, i_{G}\right)$ oscillation imposed by the vertical tide. The top panel (a) compares the model with only tides to the one with only stars, and the grey dots are individual stellar injections. In the bottom panel (b) the stars-only model is replaced by the combined model

method to use for the Galactic tide perturbation during the coming orbital period. The fastest method is a mapping (Breiter et al. 2007) that analytically computes the orbital elements at the subsequent perihelion, but this is used only for elliptic orbits with semi-major axis less than a critical value that depends on the eccentricity (Fouchard et al. 2007). Otherwise we use numerical integration with a symplectic integrator for KS-regularized equations of motion (Laskar and Robutel 2001) in case $1 / a>10^{-5} \mathrm{AU}^{-1}$ and the 15th order RADAU integrator (Everhart 1985) for $1 / a<10^{-5} \mathrm{AU}^{-1}$. 
During the orbital period in question, normally, several stellar passages occur. On those occasions the osculating cometary orbit is subject to an instantaneous impulse. In the numerical integration regime for the Galactic tide, one always comes back to perihelion. But in the mapping regime, when the starting orbital period has elapsed, the comet may not be at perihelion because of the intervening stellar perturbations, and we then resort to numerical integration until the next perihelion passage takes place.

The simulation proceeds for a maximum of $5 \mathrm{Gyr}$, unless an end state is reached. There are two such end states: either the comet reaches perihelion with $q<q_{c}=15 \mathrm{AU}$ (it is lost due to planetary perturbations), or the comet reaches $r=4 \times 10^{5} \mathrm{AU}$ in outbound motion (it escapes directly into interstellar space).

What we have just described is the full simulation of the "combined" model including both the Galactic tide and stellar perturbations. In addition, we have run two simulations that include only one or the other of the two dynamical effects.

\section{Injection time}

We will first consider the time needed to shift any cometary starting perihelion distance $q_{o}>32 \mathrm{AU}$ into a perihelion distance $q<15 \mathrm{AU}$. This is the time $t_{\text {inj }}$ required to inject a comet into the target zone, and we call it the injection time. We have thus scrutinized all three simulations, and for each injected comet in every simulation we noted its value of $t_{\text {inj. }}$. Let us now compare the statistics of injection times between all three dynamical models.

The range of initial semi-major axes $3000<a_{o}<100000 \mathrm{AU}$ is divided, according to $\log a_{o}$, into ten equal intervals. For each interval the following statistical parameters concerning the injection time are computed: its median value, its lower and upper quartiles (surpassed by $75 \%$, respectively $25 \%$, of the values), its lower and upper deciles (surpassed by $90 \%$, respectively $10 \%$, of the values), and finally its lower and upper percentiles (surpassed by $99 \%$, respectively $1 \%$, of the values).

Figure 1 presents the comparison of $t_{\text {inj }}$ statistics by means of two plots. The top one (Fig. 1a) compares the model with only the Galactic tides to the combined model, while in the bottom one (Fig. 1b) the tides-only model is compared to the one with only stellar perturbations. In each case we plot the statistical quantities versus $a_{o}$. The tides-only model is represented by filled squares, and for the other models we use half-filled circles. At any particular value of $a_{o}$, the symbols for each model are joined by vertical bars. A slight horizontal shift between the symbols has been introduced for easy distinction of the models, but the real $a_{o}$ intervals are identical. The median values have been joined by curves (dotted for the tides-only model, and solid for the other ones). The grey dots show individual injections for the stars-only model (Fig. 1b) and the combined model (Fig. 1a).

For comparison with Duncan et al. (1987), who plotted a similar diagram (their Fig. 2), we include three lines. The one labelled $T_{g}$ shows their "tidal torquing time" and the one labelled $T_{s}$ shows the corresponding "stellar torquing time", both as functions of $a$. These are meant to represent the typical time required to decrease the perihelion distance from $25 \mathrm{AU}$ to $15 \mathrm{AU}$ in the two cases. The third line labelled $T_{r}$ refers to the period of $\left(q, i_{G}\right)$ oscillation due to the tidal component normal to the Galactic plane (Duncan et al. 1987).

By inspecting Fig. 1, we can make the following observations. First, compare the tidesonly median curve with the $T_{r}$ line. The two agree fairly well in the range from $a \simeq 6000$ to $25000 \mathrm{AU}$. This is natural, because $T_{r}$ is twice the average time it takes for the vertical tide to bring any Oort Cloud comet into the target zone, as long as it is on a relevant trajectory with $q_{\min }<15 \mathrm{AU}$. For $a \lesssim 6000 \mathrm{AU}$ the median flattens out at about $2.5 \mathrm{Gyr}$, and this 


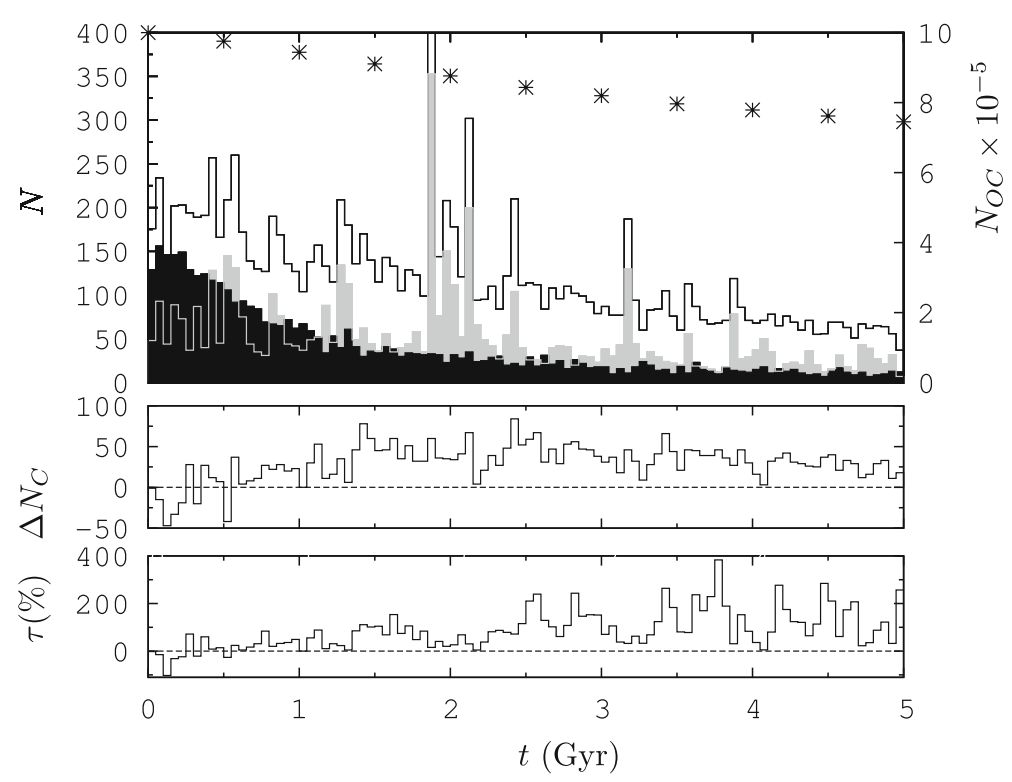

Fig. 2 The upper diagram shows the number of comets entering the observable zone per 50 Myr versus time. The white histogram corresponds to the combined model, the black histogram to the Galactic tide alone, and the grey histogram to the passing stars alone. The asterisks indicate the number of comets remaining in our simulation for the combined model at every $500 \mathrm{Myr}$ with scale bars to the right. The middle diagram shows the excess number of injections into the observable region per $50 \mathrm{Myr}$ in the combined model with respect to the sum of the stars-only and tides-only models. The lower diagram shows this excess expressed in percent of the mentioned sum

is due to the limit of our simulated interval at $5 \mathrm{Gyr}$. Had we let the simulation run for a much longer time, we would have seen the median curve follow the $T_{r}$ line to even smaller $a$ values. For $a \gtrsim 25000 \mathrm{AU}$ we see how the median curve turns upwards, while $T_{r}$ continues to decrease. This can be explained as a result of a quick stripping of comets from all trajectories with $q_{\min }<15 \mathrm{AU}$, after which these have to be repopulated through the action of the non-integrable part of the tides. Since this works on a much longer time scale, it is obvious that the median of $t_{\text {inj }}$ has to increase.

Already at this point we see evidence that the mean injection time-even in the tidesonly model—does not follow the prediction of the $T_{r}(a)$ dependence at all semi-major axes. Studying the Oort Cloud over a long enough time allows other parts of the Galactic tide than the simple, vertical component to take control of comet injections, at least in the outer parts of the cloud. But consider now the median curves of the two models that involve stellar perturbations. They are mutually quite similar, but they differ strongly from the tides-only curve except at $a \lesssim 6000 \mathrm{AU}$.

The mutual similarity-in spite of a much larger number of grey points (injections) in Fig. 1b-means that the same basic mechanism is at work. We identify this as the angular momentum reshuffling by stellar perturbations. In Fig. 1a (stars-only model) this in itself makes comets diffuse all over angular momentum space so that some reach the target zone. In Fig. 1b (combined model) the same angular momentum diffusion repopulates the "infeed trajectories" (with $q_{\min }<15 \mathrm{AU}$ ) of the vertical Galactic tide, whereupon the comets are injected at a rate given by $T_{r}(a)$. We interpret the flatness of the median curves at a level of roughly half the duration of our simulation as evidence that the time scale of angular 
momentum reshuffling is short enough to guarantee an injection rate that is at least as large during the first half $(0-2.5 \mathrm{Gyr})$ as during the second half $(2.5-5 \mathrm{Gyr})$. This is in agreement with the thermalization time scale reported by Duncan et al. (1987). The tendency for a slight decrease of the median $t_{\text {inj }}$ at the largest semi-major axes is likely due to a progressive depletion of the outer parts of the cloud during the course of the simulation, while the reshuffling time scale is relatively short.

We thus realize that the behaviour of the median injection time, generally speaking, has very little to do with any of the theoretical time scales. Let us now instead consider the lowest percentiles, since these give information on the quickest injections that-in principle-might be governed by the $T_{g}$ or $T_{s}$ dependences. The lowest percentile of the tides-only model indeed decreases with $a_{o}$ roughly parallel to the $T_{g}$ line in the inner core of the cloud ( $a<10000 \mathrm{AU}$ ), but we nonetheless see a somewhat smaller slope. This tendency gets stronger with increasing $a_{o}$ and finally turns into an increase outside $30000 \mathrm{AU}$. We interpret this as due to the same repopulation of infeed trajectories by the non-integrable part of the tide as we discussed in relation to the median curve.

The lowest percentile of the stars-only model shows a fall-off with $a_{o}$ that is interestingly slow in comparison with the $T_{s}$ line. This appears to be related to the horizontal bands of grey points, which are cometary showers. For each $a_{o}$ interval in the inner core, the timing of the lowest percentile is that of the first shower reaching into that interval. The larger $a_{o}$, the sooner such a shower appears. But the showers also get weaker, being caused by more and more distant stellar encounters. Thus, in the outer parts of the cloud they are no longer of significance for defining the lowest percentile. Since this is instead controlled by a growing number of usual, inefficient stars passing through the outer regions, one has to wait longer.

When we look at the lowest percentile of the combined model, we see that it follows the same decrease as the tides-only model in the inner core. Indeed, with a much larger number of injections, the comet showers have lost their importance, and as we shall see in Sect.4, during the first Gyr the injections are largely controlled by the Galactic tides. But outside the inner core the lowest percentile now behaves with respect to that of the tides-only model in a similar way as the median does, and the reason is the same. Going to larger semi-major axes, in both models we see an increasing number of late injections, although for different reasons, and these determine the behaviour of most statistical parameters, causing them to decrease less rapidly than the $T_{g}$ line.

In summary we can state that we have found the theoretical time scales of Duncan et al. (1987) to give a rather poor representation of our statistics of injection times. On the other hand, we find evidence in the combined model for an important role being played by the repopulation of tidal infeed trajectories via stellar encounters-something that may be described as a synergy effect. This being said, one nonetheless realizes that $T_{r}$ is one of the basic time scales that govern this synergetic injection, the other one being the angular momentum reshuffling time scale of stellar perturbations. Let us now move to a discussion of the rate of injections and how this depends on time.

\section{Time dependent injection flux}

The upper part of Fig. 2 shows a histogram plot of the number of comets injected into the observable region as a function of time from the beginning till the end of the simulation. Three histograms are shown together: the one in black corresponds to a model with only Galactic tides, and the grey one to a model including only stellar perturbations. Finally, the top, white histogram is for the combined model that includes both tides and stars. 
The first thing to note is the gradual decline of the injection flux of the tides-only model over a few Gyr, after which it stays at a very low level. The reason is clear. In the beginning, the phase space trajectories that in the regular dynamics imposed by the vertical tide will periodically lead into the "loss cone" $(q<15 \mathrm{AU})$ are populated just as densely as any phase space domain and thus furnish an important flux of injections during the first period of $\left(e, i_{G}\right)$ oscillations. This amounts to a typical time scale of $<1 \mathrm{Gyr}$ for much of the initial cloud, but the population on these trajectories is depleted by each entry into the loss cone, and there is no efficient way to replenish them without including stellar passages. On the longer time scale, we see only the feeble flux coming from (1) the infeed into the tidal injection trajectories by the non-integrable part of the tide; (2) the inner parts of the cloud, where the period of oscillation is very long (Fouchard et al. 2006).

The other two histograms include the effects of stellar passages, and the stars are the same in both simulations. Therefore, we see the same comet showers appearing and the same quasi-quiescent periods in between. The white area at the top of each bin corresponds to the extra contribution of the combined model as compared with that of the stars only. If the numbers plotted in the white, grey and black histograms are called $N_{C}, N_{S}$ and $N_{G}$, respectively, we can define $\Delta N_{C}=N_{C}-N_{S}-N_{G}$ as an absolute measure of this extra contribution. ${ }^{1}$ Already at first glance, looking at the later part of the simulation, we see that this is very significant. In the two lower panels of the figure, we plot histograms of $\Delta N_{C}$ and $\tau=\Delta N_{C} /\left(N_{S}+N_{G}\right)$, i.e., the extra contribution expressed as a fraction of $N_{S}+N_{G}$.

The basic observations are as follows. The spiky nature of the grey histogram is due to comet showers caused by close stellar encounters (we will briefly treat these below). While during the first Gyr the level of $N_{G}$ is generally higher than that of $N_{S}$, this situation gets reversed after more than 2 Gyr. Even outside the main showers, $N_{S}$ is then at a somewhat higher level than $N_{G}$. The white histogram, showing $N_{C}$, shares the spikes of the strongest showers, but the contrast between the spikes and the background is less than in the grey histogram. Indeed, the $\Delta N_{C}$ histogram shows no spikes at all, and the general level does not seem to correlate with the stellar injection rate, as illustrated by Fig. 3. Therefore, during the later part of the simulation, the $\tau$ parameter shows fluctuations anticorrelated with those of $N_{S}$. It reaches a few hundred percent, when $N_{S}$ drops to its lowest levels, but sometimes decreases to nearly zero during the peaks of $N_{S}$.

In order to smooth out those fluctuations we present in Table 2 time averages of $\tau$ over 1 Gyr periods along with the corresponding integrals of $N_{C}, N_{S}$ and $N_{G}$. During the first Gyr the flux of the combined model is not much larger than the sum of the two fluxes with separate effects, and the difference is just a small fraction of the total flux. But toward the end the synergy effect of the combined model, as measured by $\Delta N_{C}$, has grown-on the average - to nearly the same level as $N_{S}+N_{G}$. During the last Gyr we find that $\left\langle N_{C}\right\rangle$ is about 2.5 times larger than $\left\langle N_{S}\right\rangle$ in fair agreement with earlier estimates by Heisler et al. (1987) and Heisler (1990). After an initial, relatively fast decrease due to the emptying of the tidal infeed trajectories, $\left\langle N_{C}\right\rangle$ continues to decrease approximately in proportion to the total number of Oort Cloud comets $\left(N_{O C}\right)$, and $\left\langle N_{S}\right\rangle$ and $\left\langle N_{G}\right\rangle$ show similar behaviours.

Looking in detail at the $\Delta N_{C}$ and $\tau$ histograms in Fig. 2 for the beginning of our simulation, we see that they start from negative values and turn into positive ones after $\sim 0.5 \mathrm{Gyr}$. Thus, in the very beginning, the sum of the separate fluxes is larger than the combined flux. This phenomenon was found by Matese and Lissauer (2002), whose calculations were limited

\footnotetext{
${ }^{1}$ Towards the end of our simulation the number of Oort Cloud comets has decreased in all three models but most in the combined one. We then have about 930000,840000 and 760000 comets in the tides-only, starsonly and combined models, respectively. This means that $\Delta N_{C}$ actually underestimates the extra contribution of the combined model.
} 
Fig. $3 \Delta N_{C}$ versus $N_{S}$ (plotted on a $\log$ scale), where the numbers refer to injections of comets into the observable region during intervals of $10 \mathrm{Myr}$

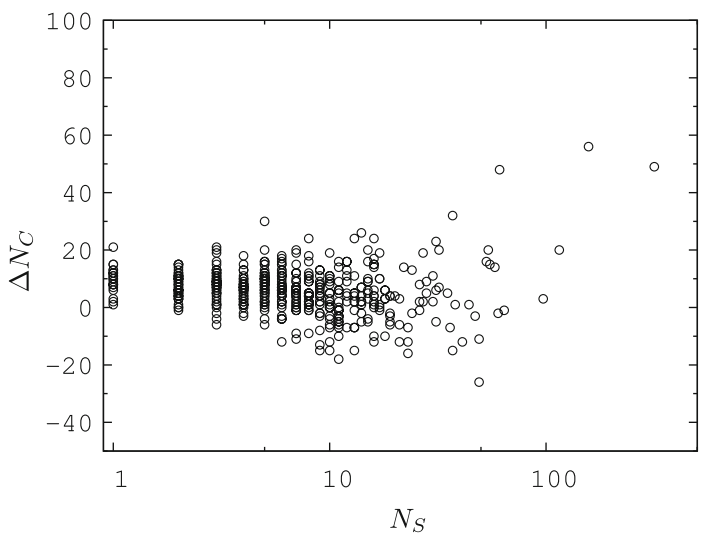

Table 2 Number of comets entering the observable region during periods of $1 \mathrm{Gyr}$

\begin{tabular}{llllll}
\hline Model & {$[0-1] \mathrm{Gyr}$} & {$[1-2] \mathrm{Gyr}$} & {$[2-3] \mathrm{Gyr}$} & {$[3-4] \mathrm{Gyr}$} & {$[4-5] \mathrm{Gyr}$} \\
\hline $\mathrm{G}$ & 2128 & 797 & 481 & 307 & 248 \\
$\mathrm{~S}$ & 1425 & 1555 & 1030 & 717 & 511 \\
$\mathrm{C}$ & 3618 & 3141 & 2,412 & 1733 & 1274 \\
$\langle\tau\rangle$ & $1.8 \%$ & $33.6 \%$ & $59.6 \%$ & $69.2 \%$ & $67.9 \%$ \\
\hline
\end{tabular}

Model $\mathrm{G}$ corresponds to the Galactic tide alone, $\mathrm{S}$ to passing stars alone, and $\mathrm{C}$ to Galactic tide and passing stars together. $\langle\tau\rangle$ is the increment from the sum of the two first rows (Galactic tide plus passing stars separately) to the third row (Galactic tide and passing stars together)

to only $5 \mathrm{Myr}$, and as they explained, it is typical of a situation where both tides and stars individually are able to fill the loss cone to a high degree. We will discuss this point again in Sect. 5 .

The large amount of synergy ( $\tau \sim 70 \%$ ) seen in the later part of our simulation is remarkable and indicates that both the tides and the stars on their own are seriously inefficient in filling the loss cone. It is only by means of the synergy of both effects that we are able to explain an important degree of loss cone filling at the current epoch. We will look at this closer in Sect. 5 by separating the injection flux into different ranges of semi-major axis. For the moment we emphasize that treating comet injections from the Oort Cloud in the contemporary Solar System simply as a result of the Galactic tide is not a viable idea.

Already in Sect. 3 we identified a mechanism that offers a likely explanation of the synergy effect, i.e., the repopulation of tidal infeed trajectories via stellar encounters. But note in Fig. 2 that the initial flux of the model with tides only is not matched by the white areas in the later part of the simulation. Thus, even though there is an ongoing replenishment of the tidal infeed trajectories due to the randomizing effect of stellar encounters, this replenishment is not complete. The critical trajectories remain largely depleted, and models that do not take this fact into account will overestimate the tidal contribution to the injection flux, as well as the efficiency of tides in filling the loss cone.

The most important synergy mechanism of the Galactic tide and stellar perturbations is that the latter are able to repopulate the critical phase space trajectories that in the quasiregular dynamics imposed by the tide lead into the loss cone (Dybczyński 2002; Fernández 2005). Our results appear to verify and quantify this picture. But in addition we see hints that a different effect is also at work. In Sect. 5 we will show that the distribution of $1 / a$ 
of newly injected, observable comets-even during the typical, quiescent periods - has a significant extension inside the limit (at $a \simeq 3 \times 10^{4} \mathrm{AU}$ ), where the tide becomes able to feed comets from outside the loss cone into observable orbits. Our explanation for this effect is as follows.

In qualitative terms, when the Galactic tide is in the process of injecting a comet into the observable region from the region outside the loss cone, and stellar perturbations are added, the latter will sometimes aid in decreasing the perihelion distance of the comet $\left(\Delta q_{*}<0\right)$, and sometimes they will counteract the tide $\left(\Delta q_{*}>0\right)$. To first order, the two effects will cancel. But if we consider how much the critical value $\left(1 / a_{c}\right)$ of the inverse semi-major axis for tidal injection into the observable region $\left(a_{c} \simeq 3 \times 10^{4} \mathrm{AU}\right)$ is decreased by a typical positive $\Delta q_{*}$ or increased by the same typical value of $\Delta q_{*}$ in the negative direction, we find that the latter effect dominates. Thus, a negative $\Delta q_{*}$ causes a larger gain of comets with $a<a_{c}$ than the loss of comets with $a>a_{c}$ caused by a positive $\Delta q_{*}$ of the same size. This holds for any nearly uniform distribution of $1 / a$ in the Oort Cloud.

In mathematical terms, consider the expression for the maximum possible decrease of $q$ in one revolution due to the Galactic tide:

$$
\Delta q=-S q^{1 / 2} z^{-7 / 2}
$$

where $z=1 / a$ and $S=2.8 \times 10^{-15}$ (Byl 1986), counting $q$ and $a$ in AU. This would hold for a Galactic latitude of perihelion of $\pm 45^{\circ}$. We take this favourable orbital orientation as an example, but the following arguments apply for any other orientation as well.

Next, consider a particular value $\left(q_{p}\right)$ of the perihelion distance preceding the injection into an observable orbit. Using Eq. 2, one can write down an approximate condition for the critical value $z=z_{0}$ in order to bring the comet into the observable region $\left(q<q_{\ell}=5 \mathrm{AU}\right)$ :

$$
q_{p}=q_{\ell}+S q_{p}^{1 / 2} z_{0}^{-7 / 2}
$$

Equation 3 defines a unique relation between $q_{p}$ and $z_{0}$, and by differentiating one easily finds that $q_{p}$ decreases monotonously with $z_{0}$, while the second derivative is always positive.

Considering thus an arbitrary point $\left(z_{0}, q_{p}\right)$ satisfying Eq.3, we may introduce stellar perturbations by adding a term $-\Delta q_{*}$ to the right-hand member of Eq. 3, and we can write:

$$
q_{p}=q_{\ell}+S q_{p}^{1 / 2} z_{1}^{-7 / 2}-\Delta q_{*}
$$

where $z_{1}$ is the new critical value of $z$. Hence, $\left(z_{1}, q_{p}+\Delta q_{*}\right)$ also satisfies Eq.3. Without the stellar perturbation all comets with $q=q_{p}$ and $z<z_{0}$ are injected into $q<q_{\ell}$, and including the stellar perturbation, the condition changes into $z<z_{1}$. From the negative slope of the $q_{p}\left(z_{0}\right)$ relation it is obvious that a negative value of $\Delta q_{*}$ yields $z_{1+}>z_{0}$, and the same positive value yields $z_{1-}<z_{0}$. It is also obvious from the positive curvature of the graph that $z_{1+}-z_{0}>z_{0}-z_{1-}$. If $\left|\Delta q_{*}\right|$ is very small, this difference is negligible, but if it is large enough to compete with $\left|\Delta q_{G}\right|$, the effect will be important. The latter is indeed often the case, when we discuss injections from just outside the loss cone ( $\left.q_{p} \simeq 15 \mathrm{AU}\right)$, as has been shown, e.g., by Duncan et al. (1987) and Fernández (2005). Therefore, the gain of comets with $z>z_{0}$ occurs over a larger interval than the loss with $z<z_{0}$ for a symmetric distribution of stellar perturbations.

Another issue is the distribution function of $z$ for the Oort Cloud. Our simulations start with a probability density $f(z) \propto z^{-1 / 2}$ as appropriate for an Oort Cloud formed according to the model of Duncan et al. (1987). In such a situation there would be more comets per unit interval of $z$ at $z<z_{0}$ than at $z>z_{0}$, and the gain effect would be counteracted and possibly turned into a net loss of injected comets. 
However, an interesting result of our simulations is that the gradual loss of comets from the Oort Cloud changes the distribution of $1 / a$. In agreement with a recent study by Brasser et al. (2008), we find that the loss of comets from the outer parts of the cloud is not compensated by diffusion from the inner parts, so that after $4.5 \mathrm{Gyr}$, when the number of comets has decreased from $1 \times 10^{6}$ to $7.6 \times 10^{5}, f(z)$ has become roughly flat over the range from $a=20000$ to $100000 \mathrm{AU}$. This shows that we have to expect a net gain of newly injected, observable comets resulting from a synergy of $\Delta q_{G}$ and $\Delta q_{*}$. Moreover, there should be a shift of comets from outside to inside the tidal injection limit—probably explaining why we see a significant flux of new comets all the way down to $a \simeq 20000 \mathrm{AU}$.

Although we cannot provide exact numbers, it appears that the secondary synergy mechanism due to what we may call "constructive interference" of the two effects-even though it certainly exists-is not the dominating one. The tentative evidence comes from the relative smoothness of the $\Delta N_{C}$ histogram (Fig. 2) and the lack of correlation between $\Delta N_{C}$ and $N_{S}$ (Fig. 3). These properties are expected of the repopulation of tidal infeed trajectories because of the long response time ( $\sim$ several $10^{8}$ years) for tidal infeed on the $T_{r}$ time scale (Fig. 1$)$. But if the constructive interference had been very important, we would have expected $\Delta N_{C}$ to increase immediately upon an increase of $N_{S}$-with the caveat that visible peaks of our $N_{S}$ histogram might arise primarily from a temporary infeed of inner Oort Cloud comets with $z>>z_{o}$, which do not contribute to the interference. Further detailed studies are needed to clarify this issue.

The cometary showers, displayed in Fig. 4 by means of a histogram of the injection flux of the combined model with a bin width of only $10 \mathrm{Myr}$, are seen to be quite important for the injection of comets from the Oort Cloud, as expected and as found by other authors previously (e.g., Heisler et al. 1987). We are saving a detailed analysis of those for a later paper. At present, we can only remark that the results presented here are hard to compare with the treatments of cometary showers by Heisler et al. (1987) or Heisler (1990). The first of these papers treated only comets with $a=10000$ or $20000 \mathrm{AU}$ with a full dynamical model and then only for a time interval of less than $200 \mathrm{Myr}$. The second gave only a brief account of a simulation for $4.5 \mathrm{Gyr}$ and then only for injections from $q>10$ to $q<10 \mathrm{AU}$ instead of our requirement that the perihelion has to fall substantially from $q>15$ to $q<5 \mathrm{AU}$.

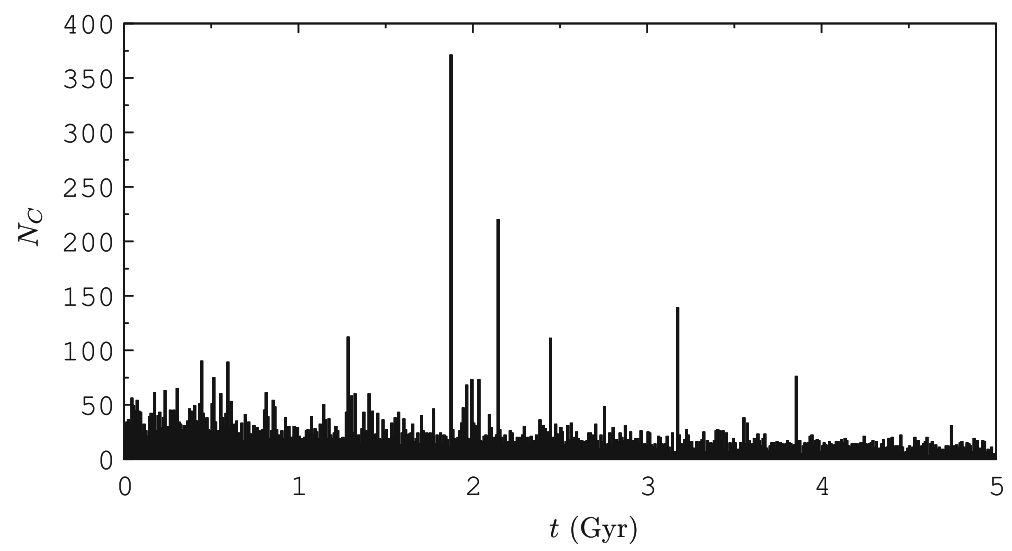

Fig. 4 Same as Fig. 2 (upper panel), but versus time per 10 Myr for the combined model only 


\section{Orbital element distributions of observable comets}

Figure 5 shows the distributions of the opposite of the inverse semi-major axis $(-1 / a)$ and the sine of the Galactic latitude of perihelion (for clarity we use the absolute value $|\sin b|$ ) of the comets entering the observable region, i.e., heliocentric distance smaller than $5 \mathrm{AU}$, during a typical $170 \mathrm{Myr}$ interval near the end of our simulation, where no strong comet showers are registered. We show an average of three such periods, i.e., 4.38-4.55 Gyr, 4.55-4.72 Gyr and 4.80-4.97 Gyr. In fact, comparing the three data sets, we find a rather good agreement, so that tentatively, the expected error of the mean is not very large. Three histograms are shown for each quantity: the one in black is for the model with Galactic tides only, the grey one is for the model with only stellar perturbations, and the white one shows the combined model.

After more than $4 \mathrm{Gyr}$ the Galactic tides alone are practically only able to inject comets into the observable region if $a>50000 \mathrm{AU}$, so that the non-integrable part of the tides may provide new comets into the emptied infeed trajectories of the vertical component. Thus the feeble flux of new observable comets is strictly confined to the outermost parts of the Oort Cloud. If only the stellar perturbations are at work, the injected comets are almost as few as in the case of the Galactic tides. However, the distribution of $-1 / a$ shows that the stellar perturbations are relatively efficient injectors of comets with semi-major axes in the whole range from 25000 to more than $100000 \mathrm{AU}$, and there is some marginal infeed all the way into the inner core. Note that this concerns a time interval without any strong comet showers.

When both the processes are at work, the number of comets entering the observable zone is 206 , about $86 \%$ more than the sum of the two separate contributions $(39+72)$. This estimate of $\tau$ is a bit higher than for the entire $1 \mathrm{Gyr}$ interval, listed in Table 2, because the three intervals have been selected as particularly calm, avoiding even the smaller peaks of $N_{S}$ that can be seen in Fig. 2. We have shown above that larger values of $N_{S}$ lead to smaller values of $\tau$. The distribution of $-1 / a$ is as wide as for the stellar perturbations alone. However, the picture has changed, since the additional $86 \%$ of the comets are strongly concentrated to the
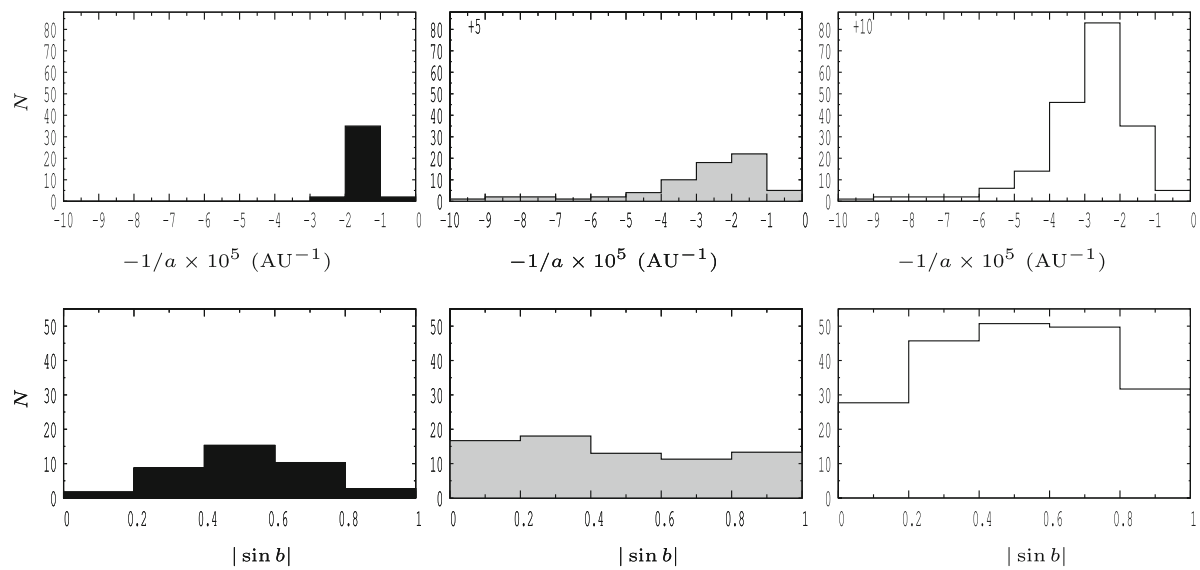

Fig. 5 Distributions of $-1 / a$, where $a$ is the semi-major axis (top panels) and $|\sin b|$, where $b$ is the Galactic latitude of perihelion (bottom panels), for the comets entering the observable region during $170 \mathrm{Myr}$ near the end of the simulation. When present, numbers in the top-left corners of $-1 / a$ distribution panels correspond to comets with $-1 / a<-1 \times 10^{-4} \mathrm{AU}^{-1}$. The left column corresponds to the model with Galactic tide alone, the middle column to passing stars alone, and the right column to the model with both effects 
interval from $-4 \times 10^{-5}$ to $-2 \times 10^{-5} \mathrm{AU}^{-1}(25000<a<50000 \mathrm{AU})$. The local values of $\Delta N_{C}$ for the five $1 / a$ intervals (0-1), (1-2), (2-3), (3-4) and (4-5) $\times 10^{-5} \mathrm{AU}^{-1}$ are -2 , $-22,+63,+36$ and +10 , respectively. We will comment on the negative values of the first entries in connection with Fig. 7.

We see that the mechanism of synergy that increases the flux of injections in the combined model prefers the range of semi-major axis $(a>30000 \mathrm{AU})$ where the vertical Galactic tide is able to provide the injections, once the relevant trajectories are populated. But there is an important extension of the synergy to smaller semi-major axes as well, extending at least to $a \simeq 20000 \mathrm{AU}$. We conclude that both the above-described synergy mechanisms must be at work. The repopulation mechanism is obviously important, but the shift to smaller semi-major axes can only be explained by the 'constructive interference' mechanism.

Looking at the distributions of $|\sin b|$, indeed the signature of the Galactic tide is clearly present in the left diagram and absent in the middle one. However, it appears again to some extent in the right-hand diagram, where the combined model is presented. Thus we have evidence that the synergetic injection of comets in the combined model carries an imprint in the latitudes of perihelia similar to that of the Galactic tide, though the feature is strongly subdued. In fact, while the subdued tidal imprint is consistent with an important role being played by the 'constructive interference' synergy mechanism, our combined model does not appear to reproduce the observed $|\sin b|$ distribution of new Oort Cloud comets. An in-depth study of this problem and a consideration of ways out of this possible dilemma are deferred to future papers.

The shaping of the $b$ distribution by the Galactic tide was first simulated numerically for a realistic Oort Cloud model by Matese and Whitman (1989). However, the left panels of Fig. 5 show a behaviour that is in stark contrast to their results. Practically all our tidal injections occur for $a>50000 \mathrm{AU}$, where Matese and Whitman (1989) found no tidal imprint in the $b$ distribution because of complete loss cone filling independent of $b$. In the light of our results this can be seen as an artefact of their assumption of complete randomization of the Oort Cloud orbit distribution. Indeed, as we shall find below (Table 4), the tidal loss cone filling for $50000<a<100000 \mathrm{AU}$ towards the end of our simulation is far from complete, and therefore we see the imprint of the tide in our $b$ distribution.

In Fig. 6 we show the corresponding distribution of $-1 / a$ and $|\sin b|$ for the 10 Myr interval from 3.85 to $3.86 \mathrm{Gyr}$, where Fig. 4 shows that the number of observable comets has a high peak due to a moderately strong shower. Occurring near the middle of the period, this shower dominates the time-integrated injection rate. The trigger is a M5 star with an impact parameter $d \simeq 2000 \mathrm{AU}$ and a velocity $v_{\star} \simeq 18 \mathrm{~km} / \mathrm{s}$.

The mid and right-hand $-1 / a$ distributions show that, as soon as stars are involved, the injection of comets now extends over the whole cloud, including an important fraction from the inner core with $a<10000 \mathrm{AU}$. In fact, the synergy effect is now very strong in the range from 10000 to $20000 \mathrm{AU}$, amounting to $\tau>150 \%$. This is unexpected on the basis of both the above-mentioned mechanisms, since we are discussing orbits too far inside the tidal injection limit. We are instead led to hypothesize a different mechanism. In the present case we are comparing the number of comets injected by a particular, deeply penetrating star from the mentioned range of semi-major axes in the stars-only versus the combined model. In the absence of the Galactic tides it is likely that orbits with perihelia close to but outside the loss cone have been depleted by the preceding cometary showers, while in the combined model the disk tide provides a regular transfer of comets into this zone on a Gyr time scale, thus compensating for the losses. This means that the synergy works in the opposite sense compared to the normal situation outside the showers. During a shower the tides are providing the material for injections by the stars, while in the normal situation the stars are 

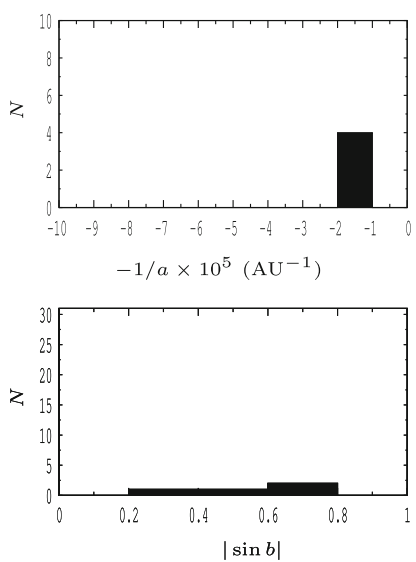

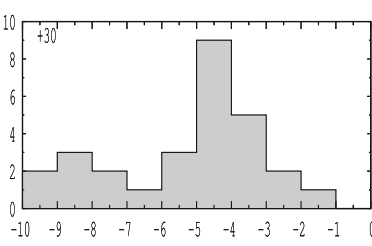

$-1 / a \times 10^{5}\left(\mathrm{AU}^{-1}\right)$
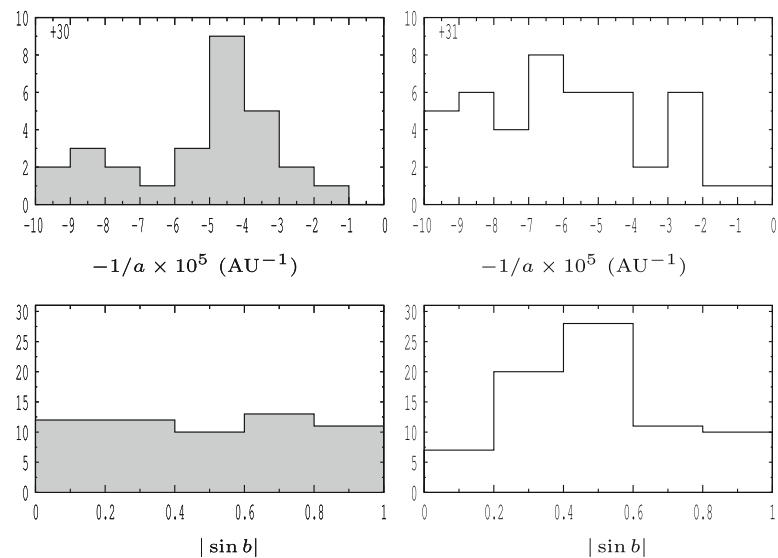

$-1 / a \times 10^{5}\left(\mathrm{AU}^{-1}\right)$

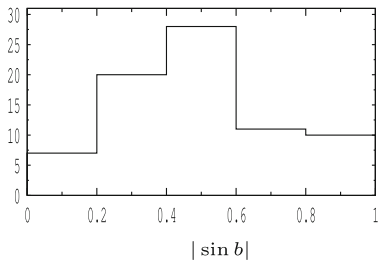

Fig. 6 Same as Fig. 5 but considering the comets that enter the observable region during a shower between 3.85 and 3.86 Gyr. The shower is due to a M5 star with impact parameter 2,055 AU, velocity $17.7 \mathrm{~km} / \mathrm{s}$ and mass $0.21 M_{\odot}$

providing the material for tidal injections. The absence of a synergy in the inner core may be explained by the very long time scale for tidal torquing, or by a smaller degree of depletion of the source region for stellar injections.

Naturally, in the stars-only model the shower does not carry any signature in the distribution of $|\sin b|$. The combined model does not exhibit any significant signature either, but there may nonetheless be a slight tendency. In case this is real, it might possibly reveal a somewhat more efficient synergy in the 10000-20000 AU range for the orbits experiencing a faster tidal decrease of $q$.

Let us now consider the situation at the beginning of the simulation, before the tides have had the time to completely empty the tidal infeed trajectories in the outer part of the cloud. The results are shown in Fig. 7 for a period between 0.63 and $0.80 \mathrm{Gyr}$, when no strong showers are noted. The number of comets entering into the observable region is 282,128 and 463 for the tides, the stars, and the combined model, respectively. The action of the tides is still quite strong, since the infeed trajectories in the interval $20000<a<50000 \mathrm{AU}$ are not yet severely depleted (cf. Fig. 1). Therefore the net synergy effect amounts to only $13 \%$.

The local $\Delta N_{C}$ values for the same five $1 / a$ intervals as we discussed in connection with Fig. 5 are in this case $-5,-66,+35,+61$ and +19 . We do not see any significant synergy effect for more tightly bound orbits. The distribution of positive synergy over the $2-5 \times 10^{-5} \mathrm{AU}^{-1}$ range is similar to that of Fig. 5, and our conclusions about the relevance of the two mechanisms are the same. Note that in both cases we see negative $\Delta N_{C}$ values in the two outermost $1 / a$ ranges $\left(0-2 \times 10^{-5} \mathrm{AU}^{-1}\right)$. The fundamental reason is the one discussed above in order to explain the negative $\tau$ values in the very beginning of the simulation, i.e., a saturation effect of the loss cone when both injection effects individually are able to cause a large degree of filling. The distributions of $|\sin b|$ exhibit the same features as in Fig. 5 and lead to the same conclusion: when both tides and stars act in synergy, the signature of the Galactic tide may be seen but appears quite marginal.

Since we noted in Sect. 4 that the replenishment of the tidal infeed trajectories by stellar perturbations is not complete during the later part of our simulation, an obvious consequence is that the filling of the loss cone for the relevant semi-major axes cannot be complete either. To quantify this statement, we consider the rate of perihelion passages $\dot{n}$ with $q<q_{0}$ as 

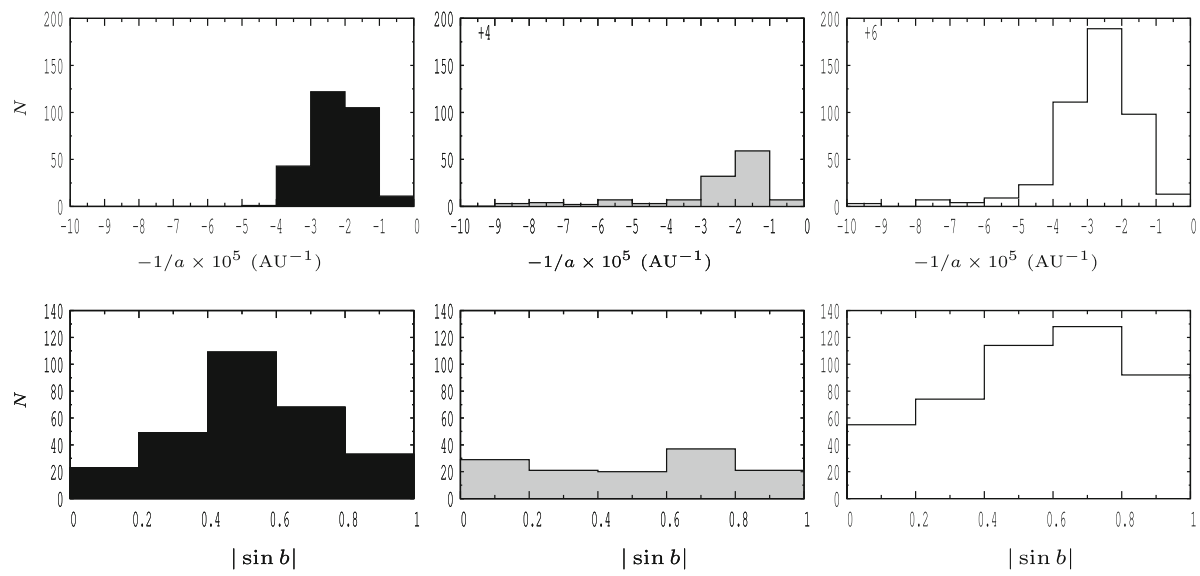

Fig. 7 Same as Fig. 5 but considering the comets entering the observable region during 170 Myr near the beginning of the simulation

a function of the semi-major axis, assuming complete loss cone filling and a completely thermalized Oort Cloud (Hills 1981; Bailey and Stagg 1988):

$$
\dot{n}(a)=N_{\mathrm{OC}} \cdot f(a) \cdot \frac{2 q_{0}}{a} \cdot a^{-3 / 2} .
$$

Here, $N_{\mathrm{OC}}$ is the number of comets in the entire Oort Cloud (initially $10^{6}$ in our model) and $f(a)$ the frequency function describing the distribution of semi-major axes: $f(a) \propto a^{-1.5}$ initially in our model.

We have computed injection rates in the combined model for the three time intervals of Figs. 5-7 using Eq. 5 and finding the integrals $\int N_{\mathrm{OC}} f(a) a^{-5 / 2} d a$ over different ranges of $-1 / a$ directly from the simulation output at neighbouring moments. The calculation of these integrals is done by simply adding the values of $a^{-5 / 2}$ of all the comets found in the relevant range. Multiplying by the length of each interval and putting $q_{0}=5 \mathrm{AU}$, we find the numbers $N_{\text {comp listed in Table } 3 .}$

The numbers of comet injections for each $1 / a$ range and each time interval can be read off from the figures, and they are listed as $N_{\text {sim }}$ in the table along with the ratios $N_{\text {sim }} / N_{\text {comp }}$, which give the filling factor of the observable part of the loss cone $\left(f_{\mathrm{lc}}\right)$. We find that this factor is close to $100 \%$ in the beginning of the simulation for $a>50000 \mathrm{AU}$ and remains $>80 \%$ for such semi-major axes even towards the end during quiescent periods. But the factor drops rapidly with decreasing $a$ to values near $2 \%$ at $a \simeq 20000$ AU. These results may be compared with those of Heisler (1990), who used a similar procedure for deriving $f_{\mathrm{lc}}$. She did not consider semi-major axes $a>40000 \mathrm{AU}$, and inside this limit we find somewhat smaller filling factors than she did, consistent with the fact that we use a lower value for the Galactic mid-plane density and somewhat higher stellar velocities.

Note that the filling factors have decreased somewhat, when we compare the final quiescent period with the initial one. Except in the outermost parts of the Oort Cloud, there is always a depletion of comets in the regions of phase space near the tidal infeed trajectories and in the vicinity of the loss cone, and this depletion grows slowly with time.

The numbers $N_{\text {sim }}$ found for the shower period are too small to be statistically useful for the outer parts of the cloud, and the filling factors listed are very uncertain. However, we see an obvious effect in the inner parts, when comparing $f_{\mathrm{lc}}$ with the corresponding values of 
Table 3 Numbers of comet injections during the time intervals of Figs. 5-7 for different ranges of inverse semi-major axis, as computed from Eq. 5 and found from our simulation of the combined model

\begin{tabular}{|c|c|c|c|c|c|c|c|c|c|}
\hline \multirow{2}{*}{$\begin{array}{l}\Delta(1 / a) \\
\left(10^{-5} \mathrm{AU}^{-1}\right)\end{array}$} & \multicolumn{3}{|c|}{ Beginning } & \multicolumn{3}{|c|}{ End, showers } & \multicolumn{3}{|c|}{ End, quiescent } \\
\hline & $N_{\text {comp }}$ & $N_{\text {sim }}$ & $f_{\mathrm{lc}}(\%)$ & $N_{\text {comp }}$ & $N_{\text {sim }}$ & $f_{\mathrm{lc}}(\%)$ & $N_{\text {comp }}$ & $N_{\text {sim }}$ & $f_{\mathrm{lc}}(\%)$ \\
\hline$(0-2)$ & 106 & 111 & $\sim 100$ & 3.2 & 2 & $\sim 60$ & 48 & 40 & 83 \\
\hline$(2-3)$ & 303 & 189 & 62 & 10 & 6 & $\sim 60$ & 160 & 83 & 52 \\
\hline$(3-4)$ & 616 & 111 & 18 & 24 & 2 & $\sim 8$ & 367 & 46 & 13 \\
\hline$(4-5)$ & 1044 & 23 & 2.2 & 44 & 6 & 14 & 692 & 14 & 2.0 \\
\hline$(5-10)$ & 15600 & 23 & 0.15 & 740 & 29 & 3.9 & 12100 & 13 & 0.11 \\
\hline$>10$ & 672000 & 6 & 0.0009 & 37200 & 31 & 0.08 & 626000 & 10 & 0.0016 \\
\hline
\end{tabular}

The ratio of simulated to computed number, expressed in percent, is also listed in each case

Table 4 Filling factors for the observable part of the loss cone, computed for different ranges of semi-major axis and separately for the three dynamical models (tides-only, stars-only, and combined)

\begin{tabular}{|c|c|c|c|c|c|c|}
\hline \multirow{2}{*}{$\begin{array}{l}\Delta(1 / a) \\
\left(10^{-5} \mathrm{AU}^{-1}\right)\end{array}$} & \multicolumn{3}{|c|}{$f_{\mathrm{lc}}($ Beginning $)$} & \multicolumn{3}{|l|}{$\underline{f_{\mathrm{lc}}(\text { End })}$} \\
\hline & Tidal (\%) & Stellar (\%) & Combined (\%) & Tidal $(\%)$ & Stellar (\%) & Combined (\%) \\
\hline$(0-1)$ & $\sim 400$ & $\sim 100$ & $\sim 200$ & $\sim 100$ & $\sim 100$ & $\sim 100$ \\
\hline$(1-2)$ & 86 & 60 & $\sim 100$ & 30 & 45 & 78 \\
\hline$(2-3)$ & 36 & 10 & 62 & 0.6 & 10 & 52 \\
\hline$(3-4)$ & 6.5 & 1.1 & 18 & - & 2.3 & 13 \\
\hline$(4-5)$ & 0.09 & 0.3 & 2.2 & - & 0.5 & 2.0 \\
\hline$(5-10)$ & - & 0.1 & 0.15 & - & 0.06 & 0.11 \\
\hline$>10$ & - & 0.0006 & 0.0009 & - & 0.0008 & 0.0016 \\
\hline
\end{tabular}

quiescent periods. The shower increases $f_{\mathrm{lc}}$ by factors $\sim 20-100$, and thus the overall flux exhibits the peak seen in Fig. 4 due to comets with $a<20000$ AU.

We have already made the remark that neither $\Delta N_{C}$ nor $\tau$ provides a fully satisfactory measure of the synergy effect, because they do not account for the difference of the number of Oort Cloud comets between different dynamical models-especially towards the end of the simulation. After $5 \mathrm{Gyr}$ the total number of comets in the combined model is only $\sim 80 \%$ of that in the tides-only model, and if we concentrate on comets with $50000<a<100000 \mathrm{AU}$ where the losses are the largest, the ratio of the two models is only $35 \%$. In order to compensate for such effects we have computed the $f_{\text {lc }}$ parameter separately for the three models and for all the ranges of $1 / a$, and we present the results in Table 4 . The time periods referred to are the quiescent periods of Figs. 5 and 7.

The outermost energy range is empty in all models, when the simulation starts, but it gets populated quickly - at least when stars are involved. We interpret the very large value of $f_{\text {lc }}$ in the tides-only model at the beginning as evidence that the radial tide has not extracted comets into this energy range in a uniform manner, so that our assumption of thermalization when deriving $N_{\text {comp }}$ is not justified. To a lesser extent this appears to be true also in the combined model, where stars have extracted many more comets. It is likely that this extraction too-at the early time in question - has not populated all the angular momenta in a thermalized fashion. However, the statistics is too poor to be confident about such conclusions. In any case, the loss cone filling is extremely efficient for all models, thus explaining the negative values of $\Delta N_{C}$.

For the next energy range we see the saturation effect again, especially in the beginning. At the end, the value of $f_{\mathrm{lc}}$ in the combined model is close to the sum of those in the other 
two models. Hence there is no apparent synergy in this case, but probably a real synergy has been concealed by the saturation effect. In any case the large negative value of $\Delta N_{C}$ results entirely from the small number of comets in the combined model, as discussed above. In the next three energy ranges $(20000<a<50000 \mathrm{AU})$ we see that $f_{\text {lc }}$ in the combined model is much larger than the sum of the two other entries, and for $a<20000 \mathrm{AU}$ the effect continues: adding the tides to the stars increases the loss cone filling by a factor $1.5-2$.

\section{Discussion and conclusions}

We have simulated the evolution of the Oort Cloud over $5 \mathrm{Gyr}$, using for initial conditions a relaxed model with a distribution of semi-major axis $f(a) \propto a^{-1.5}$ within the interval 3000 $100000 \mathrm{AU}$. This model is based on the results of simulation of Oort Cloud formation and evolution by Duncan et al. (1987). However, we do not find this to be a steady distribution. More comets are lost from the outer parts of the cloud than can be replaced from inside, so that our model cloud evolves into a distribution close to $f(a) \propto a^{-2}$-i.e., flat in $1 / a$.

Our dynamical model has two main limitations. We do not treat encounters with very massive Galactic perturbers, such as star clusters or Giant Molecular Cloud complexes, the justification being that they occur so rarely that the current Solar System is unlikely to feel the direct reverberations of any such encounter, and that even if they modify the structure of the Oort Cloud, our interest is not primarily in its dynamical history but rather in the way stars and Galactic tides currently interact when injecting observable comets.

Moreover, we do not treat planetary perturbations in any direct manner. Like most previous investigators (e.g., Heisler 1990) we use a loss cone defined by a limiting perihelion distance (in our case, $15 \mathrm{AU}$ ) outside which no planetary effects are included and inside which all comets are considered lost from the cloud through perturbations by Jupiter and Saturn. In terms of "transparency" of the planetary system (Dybczyński and Prętka 1997; Dybczyński 2005), our model is completely opaque $(P=1)$. This means that we are limiting our attention to a subset of the observed population of "new" Oort Cloud comets, i.e., those that have jumped directly from $q>15 \mathrm{AU}$ into their observed orbits with $q<5 \mathrm{AU}$. We are neglecting the rest of the population, which consists of comets that passed perihelia in the outer part of the loss cone without being perturbed away before arriving into observable orbits. We are also neglecting a possible contribution to the observed new comets by a "leakage" from the scattered disk (Levison et al. 2006). Therefore we prefer not to draw any conclusions in this paper regarding the total number of Oort Cloud comets or the exact values of the filling factors. Nor do we claim to make any prediction on the detailed shape of the $1 / a$ distribution of new Oort Cloud comets, until we have included the planetary perturbations in a realistic manner.

We have shown that the concept of tidal and stellar torquing time scales (Duncan et al. 1987) gives a very incomplete picture of the speed of comet injection, whether it may concern Galactic tides or stellar encounters. The distribution of injection times is largely shaped by other effects-like comet showers or the repopulation of the emptied infeed trajectories of the disk tide due to the non-integrable part of the tides or stellar perturbations.

We have also shown how-for semi-major axes large enough for the tide to populate observable orbits - the regions of the phase space occupied by trajectories leading into the loss cone get depleted during the first Gyr of Oort Cloud evolution. This would leave little chance for the tide to produce a significant number of observable comets at the current time, were it not for the capability of stellar perturbations to replenish the tidal infeed trajectories. 
We have indeed demonstrated that, during the later parts of our simulation, there is a very important synergy effect of the Galactic tide and stellar perturbations such that the combined injection rate is on the average $\sim 70 \%$ larger than that of the stars alone plus that of the tide alone. This synergy is strongest for semi-major axes between $\sim 20000$ and $50000 \mathrm{AU}$ but continues all the way into the inner core. During comet showers the synergy effect in the outer parts of the cloud practically disappears, but the one affecting the inner parts becomes very important.

We have identified two mechanisms for the synergy during quiescent periods in the outer parts of the Oort Cloud. One is that the stellar perturbations provide a supply of new comets that replenishes the depleted tidal infeed trajectories, and the other is that the gain of comet injections, when stellar perturbations decrease the perihelion distance, dominates over the loss caused by opposing perturbations. For the synergy of the inner cloud we hypothesize that the Galactic tides provide the material for stellar injections by slowly feeding the region of phase space in the vicinity of the loss cone. Thus, the general picture spawned by our results is that injection of comets from the Oort Cloud is essentially to be seen as a team work involving both tides and stars. It appears meaningless to rank the two effects in terms of strength or efficiency.

Indeed, for the smaller semi-major axes the Galactic tide does not dominate the injection of comets, contrary to the conclusions of Heisler et al. (1987) and Heisler (1990). ${ }^{2}$ It only contributes to a synergy with stellar perturbations, and without the stars one would not have any injections of comets with $a \lesssim 20000 \mathrm{AU}$.

The distribution of Galactic latitudes of perihelia of the observable comets exhibits a maximum for $|\sin b| \simeq 0.5$ as expected in the tides-only model, but in the combined model this feature can hardly be seen at all. The tides form part of the synergetic injection, but their imprint is largely washed out by the stellar contribution. But, likely due to the role of the tides in helping the stars to create comet showers, the pattern can be seen at least as clearly during a shower as during the quiescent periods. Therefore, it tentatively appears that the shape of the observed $b$ distribution can not be used to indicate whether we are experiencing any shower at present. However, since none of our model distributions appears to agree with the observed one, we have to defer any conclusions to future papers. It may be interesting to see, for instance, if the leakage from the scattered disk into the Oort Cloud with an ensuing direct transfer into observable new comets may alleviate the problem.

We have measured the filling of the observable part of the loss cone by comparing our simulated injection rates for different intervals of semi-major axis with the rates of observable perihelion passages ( $q<5 \mathrm{AU})$ computed for a completely thermalized distribution of cometary orbits involving a filled loss cone. The deficiency of our simulated rate likely reflects not only a lack of comets in the loss cone but a general depletion in a wider phase space region in its vicinity, as remarked by Heisler (1990). Our results can be compared with hers, and in contrast to her inference that $f_{\text {lc }}$ may level out at $\sim 60 \%$ for $a \gtrsim 30000 \mathrm{AU}$, we find an average filling factor during quiescent periods in the current Solar System, which drops steadily from $\sim 100 \%$ at $a>100000$ AU to $1 \%$ or less at $a<20000$ AU in the combined model. However, there are important differences between the two investigations, one being that she simulated a much shorter time period than we do, and in addition our parameters for the Galactic tides and stellar encounters also differ from hers.

\footnotetext{
2 The main reason for this discrepancy is that the Heisler papers considered injections into the loss conemainly by slight perturbations of $q$ across the limiting value $q_{c}=10 \mathrm{AU}$ - while we consider large jumps from $q>15 \mathrm{AU}$ into the observable region with $q<5$ AU. Interestingly, Heisler et al. (1991) commented that the injection into orbits with $a \lesssim 20000 \mathrm{AU}$ and $q<2 \mathrm{AU}$ is indeed dominated by stellar perturbations.
} 
In agreement with Weissman and Hut (1986), we find that cometary showers do not significantly increase the loss cone filling at large semi-major axes. However, near $25000 \mathrm{AU}$ there is an abrupt change into the regime of the inner cloud, where the filling factor increases by orders of magnitude during moderate to strong events. The showers of course involve direct injections by the passing stars, but the synergy with the Galactic tide is as important as during quiescent periods.

Acknowledgements We are greatly indebted to the referees of this paper, Ramon Brasser and Julio A. Fernández, for having pointed out various weaknesses in a previous version, which inspired us to exert more care in all parts of our analysis. M.F. is grateful to GDRE 224 CNRS INdAM, GREFI-MEFI and Ch. F. to the plan National de Planétologie (PNP) for financial support. For H.R., this work was supported by Grants nr. 78/06 and 119/07:1 of the Swedish National Space Board. The work of G.B.V. was supported by the contract ASI/INAF I/015/07/0.

\section{References}

Allen, C.: Astrophysical Quantities, 3rd edn. Athlone Press, London (1985)

Bahcall, J.N.: Self-consistent determinations of the total amount of matter near the sun. Astrophys. J. 276, 169-181 (1984)

Bailey, M.E., Stagg, C.R.: Cratering constraints on the inner Oort cloud-steady-state models. Mon. Not. R. Astron. Soc. 235, 1-32 (1988)

Brasser, R., Duncan, M.J., Levison, H.F.: Embedded star clusters and the formation of the Oort Cloud III: the Galactic phase. Icarus (in press) (2008)

Breiter, S., Fouchard, M., Ratajczak, R., Borczyk, W.: Two fast integrators for the Galactic tide effects in the Oort cloud. MNRAS 377, 1151-1162 (2007)

Byl, J.: The effect of the Galaxy on cometary orbits. Earth Moon Planets 36, 263-273 (1986)

Charnoz, S., Morbidelli, A.: Coupling dynamical and collisional evolution of small bodies II. Forming the Kuiper belt, the scattered disk and the Oort cloud. Icarus 188, 468-480 (2007)

Delsemme, A.H.: Galactic tides affect the Oort cloud-an observational confirmation. Astron. Astrophys. 187, 913-918 (1987)

Duncan, M., Quinn, T., Tremaine, S.: The formation and extent of the solar system comet cloud. Astron. J. 94, 1330-1338 (1987)

Dybczyński, P.A.: Simulating observable comets. I. The effects of a single stellar passage through or near the Oort cometary cloud. Astron. Astrophys. 396, 283-292 (2002)

Dybczyński, P.A.: Simulating observable comets. II. Simultaneous stellar and galactic action. Astron. Astrophys. 441, 783-790 (2005)

Dybczyński, P.A., Prętka, H.: The galactic disk tidal force: simulating the observed Oort cloud comets. In: Wytrzyszczak, I.M., Lieske, J.H., Feldman, R.A. (eds.) IAU Colloq. 165: Dynamics and Astrometry of Natural and Artificial Celestial Bodies, pp. 149-154. Kluwer, Dordrecht (1997)

Emel'yanenko, V.V., Asher, D.J., Bailey, M.E.: The fundamental role of the Oort cloud in determining the flux of comets through the planetary system. Mon. Not. R. Astron. Soc. 381, 779-789 (2007)

Everhart, E.: An efficient integrator that uses Gauss-Radau spacings. In: Carusi, A., Valsecchi, G.B. (eds.) ASSL Vol. 115: IAU Colloq. 83: Dynamics of Comets: Their Origin and Evolution, pp. 185-202. Reidel, Dordrecht (1985)

Fernández, J.A.: Evolution of comet orbits under the perturbing influence of the giant planets and nearby stars. Icarus 42, 406-421 (1980)

Fernández, J.A. (ed.): Comets-Nature, Dynamics, Origin and their Cosmological Relevance. Astrophysics and Space Science Library, vol. 328. Springer, Dordrecht (2005)

Fouchard, M., Froeschlé, Ch., Valsecchi, G., Rickman, H.: Long-term effects of the Galactic tide on cometary dynamics. Celest. Mech. Dyn. Astron. 95, 299-326 (2006)

Fouchard, M., Froeschlé, Ch., Breiter, S., Ratajczak, R., Valsecchi, H., Rickman, G.: Methods to study the dynamics of the Oort cloud comets II: modelling the galactic tide. In: Benest, D., Froeschlé, C., Lega, E. (eds.) Topics in Gravitational Dynamics. Lecture Notes in Physics, vol. 729, pp. 273-296. Springer, Berlin (2007)

García-Sánchez, J., Weissman, P.R., Preston, R.A., Jones, D.L., Lestrade, J.-F., Latham, D.W., Stefanik, R.P., Paredes, J.M.: Stellar encounters with the solar system. Astron. Astrophys. 379, 634-659 (2001)

Heisler, J.: Monte Carlo simulations of the Oort comet cloud. Icarus 88, 104-121 (1990) 
Heisler, J., Tremaine, S.: The influence of the galactic tidal field on the Oort comet cloud. Icarus $\mathbf{6 5}$, 13-26 (1986)

Heisler, J., Tremaine, S., Alcock, C.: The frequency and intensity of comet showers from the Oort cloud. Icarus 70, 269-288 (1987)

Heisler, J., Tremaine, S., Weissman, P., Greenberg, R: Sky distributions of Oort cloud comets during and outside of showers. Lunar and Planetary Institute Conference Abstracts, vol. 22, p. 553 (1991)

Hills, J.G.: Comet showers and the steady-state infall of comets from the Oort cloud. Astron. J. 86, 17301740 (1981)

Holmberg, J., Flynn, C.: The local density of matter mapped by Hipparcos. Mon. Not. R. Astron. Soc. 313, 209-216 (2000)

Hut, P., Tremaine, S.: Have interstellar clouds disrupted the Oort comet cloud?. Astron. J. 90, 1548-1557 (1985)

Królikowska, M.: Non-gravitational effects in long-period comets and the size of the Oort cloud. Acta Astron. 56, 385-412 (2006)

Laskar, J., Robutel, P.: High order symplectic integrators for perturbed Hamiltonian systems. Celest. Mech. Dyn. Astron. 80, 39-62 (2001)

Levison, H.F., Dones, L., Duncan, M.J.: The origin of Halley-type comets: probing the inner Oort cloud. Astron. J. 121, 2253-2267 (2001)

Levison, H.F., Duncan, M.J., Dones, L., Gladman, B.J.: The scattered disk as a source of Halley-type comets. Icarus 184, 619-633 (2006)

Matese, J.J., Lissauer, J.J.: Characteristics and frequency of weak stellar impulses of the Oort cloud. Icarus 157, 228-240 (2002)

Matese, J.J., Whitman, P.G.: The Galactic disk tidal field and the nonrandom distribution of observed Oort cloud comets. Icarus 82, 389-401 (1989)

Mazeeva, O.A.: Dynamical evolution of Oort cloud comets to near-Earth space. In: Milani, A., Valsecchi, G., Vokrouhlický, D. (eds.) Near Earth Objects, our Celestial Neighbors: Opportunity and Risk. IAU Symposium, vol. 236, pp. 43-54 (2006)

Mihalas, D., Binney, J.: Galactic Astronomy: Structure and Kinematics. Freeman, San Francisco (1981)

Oort, J.H.: The structure of the cloud of comets surrounding the solar system and a hypothesis concerning its origin. Bull. Astron. Inst. Neth. 11, 91-110 (1950)

Remy, F., Mignard, F.: Dynamical evolution of the Oort cloud. I-A Monte Carlo simulation. II-a theoretical approach. Icarus 63, 1-30 (1985)

Rickman, H.: Stellar perturbations of orbits of long-period comets and their significance for cometary capture. Bull. Astron. Inst. Czech 27, 92-105 (1976)

Rickman, H., Froeschlé, Ch., Froeschlé, Cl., Valsecchi, G.B.: Stellar perturbations on the scattered disk. Astron. Astrophys. 428, 673-681 (2004)

Rickman, H., Fouchard, M., Valsecchi, G.B., Froeschlé, Ch.: Algorithms for stellar perturbation computations on Oort cloud comets. Earth Moon Planets 97, 411-434 (2005)

Weissman, P.R.: Physical and dynamical evolution of long-period comets. In: Duncombe, R.L. (ed.) IAU Symp. 81 Dynamics of the Solar System, pp. 277-282. Reidel, Dordrecht (1979)

Weissman, P., Hut, P.: Dynamics of cometary showers. Lunar and Planetary Institute Conference Abstracts, vol. 17, pp. 935-936 (1986) 